\title{
Persistence and transmission of tick-borne viruses: Ixodes ricinus and louping-ill virus in red grouse populations
}

\author{
P. J. HUDSON ${ }^{1}$, R. NORMAN ${ }^{2}$, M. K. LAURENSON ${ }^{1}$, D. NEWBORN ${ }^{3}$, M. GAUN T ${ }^{4}$, \\ L. JONES ${ }^{4}$, H. REID ${ }^{5}$, E. GOULD 4 , R. BOWERS ${ }^{2}$ and A. DOBSON \\ ${ }^{1}$ Wildlife Epidemiology Unit, Department of Biological and Molecular Sciences, University of Stirling, \\ Stirling FK9 $4 L A$, UK \\ ${ }^{2}$ Department of Applied Mathematics, University of Liverpool, PO Box 147 Liverpool L69 $3 B X, U K$ \\ ${ }^{3}$ Upland Research Group, The Game Conservancy Trust, Crubenmore, Newtonmore, Inverness-shire PH20 $1 B E, U K$ \\ ${ }^{4}$ Institute of Virology and Environmental Microbiology, Mansfield Road, Oxford OX1 3PS, UK \\ ${ }^{5}$ Moredun Research Institute, Gilmerton Road, Edinburgh $\mathrm{EH} 17 \mathrm{7JH}, \mathrm{UK}$ \\ ${ }^{6}$ Ecology and Evolutionary Biology, Princeton University, NJ 08544-1003, USA
}

\section{SUM M A R Y}

The population dynamics of tick-borne disease agents and in particular the mechanisms which influence their persistence are examined with reference to the flavivirus that causes louping-ill in red grouse and sheep. Pockets of infection cause heavy mortality and the infection probably persists as a consequence of immigration of susceptible hosts. Seroprevalence is positively associated with temporal variations in vectors per host, although variation between areas is associated with the abundance of mountain hares. The presence of alternative tick hosts, particularly large mammals, provides additional hosts for increasing tick abundance. Grouse alone can not support the vectors and the pathogen but both can persist when a non-viraemic mammalian host supports the tick population and a sufficiently high number of nymphs bite grouse. These alternative hosts may also amplify virus through non-viraemic transmission by the process of co-feeding, although the relative significance of this has yet to be determined. Another possible route of infection is through the ingestion of vectors when feeding or preening. Trans-ovarial transmission is a potentially important mechanism for virus persistence but has not been recorded with louping-ill and Ixodes ricinus. The influence of non-viraemic hosts, both in the multiplication of vectors and the amplification of virus through non-viraemic transmission are considered significant for virus persistence.

Key words: Louping-ill, Ixodes ricinus, red grouse, co-feeding, tick-borne diseases, trans-ovarial transmission, reservoir hosts.

\section{INTRODUCTION}

The association between invertebrate vectors and disease has been recognized since biblical times although the first demonstration of vector-borne transmission was not recorded in a biting insect vector, but the ixodid tick Boophilus annulatus infected with bovine babesiosis (Smith \& Kilborne, 1893). Ticks are of considerable economic importance as vectors of infections that cause morbidity and mortality of both livestock and humans in much of the tropical and temperate regions of the world. They transmit a wide range of harmful parasites including helminths, protozoa, rickettsiae, bacteria and viruses and the ticks themselves can cause direct damage to their host. Control measures centre around reducing tick abundance through the treatment of hosts with acaricides and in some cases destruction of tick favoured habitats. While tick control and vaccination of susceptible hosts may reduce tick-borne diseases, they are both highly persistent and difficult to eradicate.

Most tick-borne diseases require the pathogen to undergo a period of obligatory development within the vector before successful transmission, making the ticks true intermediate hosts. Even so, the dynamics of vector-borne pathogens are fundamentally different from the directly transmitted pathogens. Transmission is dependent on an infectious vector biting a susceptible host and this is a function of the number of vectors biting each host rather than the number of susceptible individuals within the host population. Many vectors can only make a fixed number of bites within their life time and this is usually independent of host density. There are also differences between the dynamics of infection when pathogens are transmitted by biting insect vectors compared with those transmitted by ticks (Randolph, 1995). First, ticks operate on a much longer time-scale than dipteran vectors. Ticks frequently take several years to complete their life cycle while the mean life cycle of most insect vectors is much shorter than the life cycle of their hosts, typically a matter of days or weeks. Secondly, lateral movement of ticks when questing for a host is very limited and they are dependent on the movement of their potential vertebrate hosts into their immediate vicinity to make contact. In contrast, insect vectors are mobile covering large distances while actively seeking out potential hosts, they will often operate over a spatial scale similar to the home range of their host. Dye \& Williams (1995) looked in detail at the 
dynamics of pathogens transmitted by biting insect vectors and addressed the processes which could generate nonlinearity in transmission rate. Some of the density-dependent processes they examined, such as vector mortality increasing with infection, may relate to tick-borne infections whereas others, such as preference for feeding on infected hosts, are unlikely to be important to the dynamics of tickborne infections.

A number of features of vector-borne parasite systems influences the rate of pathogen transmission from host to tick to host: successful viraemic transmission requires a susceptible tick to feed on an infectious host, for infections to be translocated to the salivary gland of the subsequent instar and for the virus to be passed back to a susceptible host. In effect, transmission will depend on the rate at which infectious vectors successfully transmit the pathogen to the susceptible host $\left(\beta_{1}\right)$ and the rate at which the infectious hosts transmit the pathogen back to the vector $\left(\beta_{2}\right)$. The probability of host infection will increase with the number of infectious ticks that bite the host and hence will be a function of the ratio of vectors feeding on each host $\left(N_{2} / N_{1}\right)$. The mortality rate of the pathogen will depend on the mortality of the infectious hosts $\left(b_{1}+\gamma_{1}\right)$ and the vectors $\left(b_{2}+\gamma_{2}\right)$ where $1 / b_{i}$ is the life expectancy of either host or vector and $1 / \gamma_{i}$, is the period of infectiousness for either host or vector respectively. The average number of new cases of the infection is the ratio of transmission to mortality, more formally presented as the basic reproductive number of the infection $\left(R_{0}\right)$ :

$R_{0}=\frac{\beta_{1} \beta_{2}\left(N_{2} / N_{1}\right)}{\left(b_{1}+\gamma_{1}\right)\left(b_{2}+\gamma_{2}\right)}$

If we compare insect-borne infections with tickborne infections, then because insect mortality rates are relatively high, transmission rates need to be large for the pathogen to persist. In contrast, ticks are longer lived so persistence is possible with lower transmission rates. In reality, the estimate of $R_{0}$ for a tick-borne pathogen will be more complicated and depend on the transmission and mortality rate of each tick stage and for each of the infectious host species. This produces a complex estimate of $R_{0}$ with many transmission and mortality parameters. Randolph \& Craine (1995) examine this in more detail and produce a general framework for the transmission dynamics of tick borne diseases which allows a quantitative assessment of the contribution of different host species and transmission routes to the value of $R_{0}$. Nevertheless, equation (1) provides a good starting point for discussing and evaluating the variables influencing persistence and transmission.

Successful invasion of the host population by a vector-borne pathogen requires the value of the reproductive number to be greater than unity; the persistence of any outbreak then depends on this value remaining, on average, above unity. This is a simplified view and assumes that the population is relatively homogeneous with equal mixing between vectors and hosts with no seasonal effects on vector abundance. Many tick-borne pathogens exhibit highly seasonal variation in abundance with pulses of susceptible hosts being introduced into the host population, pulses of infectious ticks emerging and ticks being highly aggregated both within the host habitat and on their hosts.

Simple calculations of tick-transmitted infections can imply that persistence is not expected, even though observations of disease incidence indicate that this is clearly the case. For example, consider the transmission between bank voles of Babesia microti by the tick Ixodes trianguliceps. Randolph (1995) carefully estimated transmission rates within the system and showed that on average, between 3 and 10 ticks per day must feed on infectious hosts and become infectious for pathogen persistence and yet the actual level of vector infection was much lower, even though the infection clearly persists. While such detailed calculations have not been done for other systems, coarse estimates concur with the finding that vector numbers per host are often lower than expected from the incidence of disease and level of vector infection. Clearly we are making simplistic assumptions somewhere about pathogen transmission and mortality. In this respect, this paper examines the parameters and assumptions underlying the estimation of $R_{0}$ for vector-borne pathogens but relates these specifically to processes that influence tick-borne pathogens. We illustrate these with some of our current work on the epidemiology of the red grouse-tick-louping-ill system. This paper is not meant to be an exhaustive review but a case study of our current understanding of louping-ill with some comments and comparisons with other systems.

\section{LOUPING-ILL SYSTEM}

Louping-ill is a disease of sheep and red grouse caused by a flavivirus transmitted between hosts by the sheep tick, Ixodes ricinus. Mortality amongst grouse is high and $80 \%$ of infected birds subsequently die compared with uninfected birds (Reid et al. 1978; Hudson \& Dobson, 1991; Hudson, 1992) while the mortality in sheep is lower and variable depending on breed and previous exposure. Ticks will feed on a wide range of other hosts; on moorlands these are principally mountain hares (Lepus timidis), red deer (Cervus elaphus), roe deer (Capreolus capreolus), small mammals and a range of moorland waders. However, none of these hosts produces an appreciable viraemic response to in- 


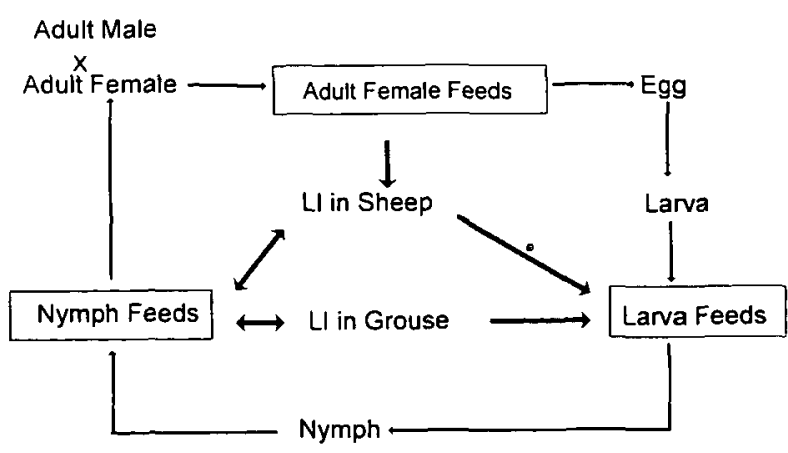

Fig. 1. Schematic representation of basic flow of louping-ill virus (LI) with respect to the tick life cycle. While ticks will bite a wide range of vertebrate host species, only sheep and grouse show an appreciable viraemic response and are represented here. There is no trans-ovarial transmission but larvae can receive the virus from infectious sheep or grouse. There is transstadial transmission so nymphs can receive or infect hosts. Adult males do not feed and females only feed on large mammals so grouse do not receive an infection from the adults. In reality, shepherds frequently vaccinate their sheep flock against the virus, so the sheep flock may not be a significant source of louping-ill.

fection other than $2 \%$ of short-tailed voles. Sheep do produce a viraemic response and on the farms where louping-ill is prevalent, shepherds will vaccinate their first year sheep (hoggs) as prevention against disease but usually leave their lambs to be protected through maternal colostrum. Initial vaccination programmes indicated the vaccine was not effective without double treatment (Shaw \& Reid, 1981), but more recent development of the vaccine has improved the efficiency of a vaccination programme. Although immunity may wane in some sheep, most are regularly challenged and immunity reinforced.

Further protection of sheep is given by regular application of acaricides. Farmers usually treat their lambs and sheep in an attempt to suppress the tick population and reduce the incidence of other tickborne diseases such as infections with Staphylococcus $\mathrm{sp}$. that cause joint-ill. In effect, the vaccination of sheep, the reinforcement of immunity and the application of acaricides remove sheep from the system and leave the grouse as the only viraemic host capable of sustaining the pathogen.

All stages of ticks (larvae, nymphs and adults) feed on mammals but only rarely are adult females found feeding on small mammals or grouse. In this respect, large mammals are essential for completing the tick life cycle and without their presence the ticks and the associated microparasites would not persist. Transovarial transmission of louping-ill in 1 . ricinus from the female, through the egg to the questing larval stage has not been detected within this system. However, there is trans-stadial transmission and once a larval tick is infected, the subsequent nymph and adult female stage are capable of transmitting virus. Since adult males do not feed and adult females do not feed on grouse, the birds will only receive the virus from an infected nymph stage. Fig. 1 summarizes the transmission of the virus in relation to the life cycle of the tick.

\section{HOST-VECTOR RATIOS $\left(N_{2} / N_{1}\right)$}

As we may expect from equation (1), variations in vector to host ratios can have large consequences on the dynamics of vector transmitted pathogens, as illustrated by studies on malaria epidemiology (Ross, 1911; MacDonald, 1957; Aron \& May, 1982; Anderson \& May, 1991). When many mosquitoes bite each host, the prevalence of malaria is stable and endemic but when few mosquitoes bite each host then slight variations in the vector to host ratio can result in substantial changes in the proportion of hosts infected and the malaria is epidemic or unstable. This pattern is dependent not only on the number of vectors biting each host $\left(N_{2} / N_{1}\right)$, but also the life expectancy of the vectors and the probability of a susceptible vector becoming infectious after biting an infectious host. Understandably, control measures that concentrate on vector control in areas of low transmission may prevent epidemic outbreaks. In contrast, in areas of high transmission, substantial changes in vector abundance may be needed to show any influence on the prevalence of the disease.

Heterogeneities in the mixing of the host population with infectious vectors can result in the basic reproductive number $\left(R_{0}\right)$ being larger than would be expected from homogeneous mixing between infectious vectors and susceptible hosts (Anderson \& May, 1991). In vector-borne pathogens, some of the largest heterogeneities are in the distribution of the vectors in relation to the host leading to spatial variations in vector to host ratios and increased estimates of the reproductive number (Dye \& Hasibeder, 1986).

To the best of our knowledge, such patterns have not been identified in tick transmitted systems. However, temporal patterns of seroprevalence of louping-ill in young grouse are related to the number of nymph ticks per host with spatial variations in grouse seroprevalence influenced by the presence of mountain hares (Fig. 2). Indeed, a spatial comparison between seroprevalence in grouse from a number of populations and environmental and biological parameters identified mountain hare abundance as the only significant factor explaining variation in seroprevalence, although this was dependent on a single point (Hudson, 1992).

\section{Seasonal variation in vector and susceptible host abundance}

Seasonal variations in the abundance of $I$. ricinus is striking. In some parts of Britain, the abundance of 


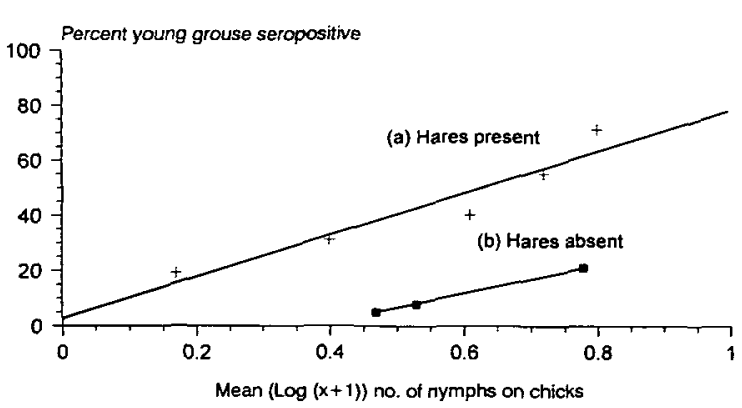

Fig. 2. The positive relationship between the annual proportion of young grouse seropositive and the mean tick burden on grouse chicks from two study areas $(a)$ Lochindorb in Scotland, with hares present and $(b)$ Westerdale in North Yorkshire where hares are absent. The probability of infection increases with tick abundance but is significantly higher in the population with hares present, even though the hares do not show an appreciable viraemic response. The abundance of alternative hosts is the major difference between these two sites. One possible confounding variable is the geographical location of the site.

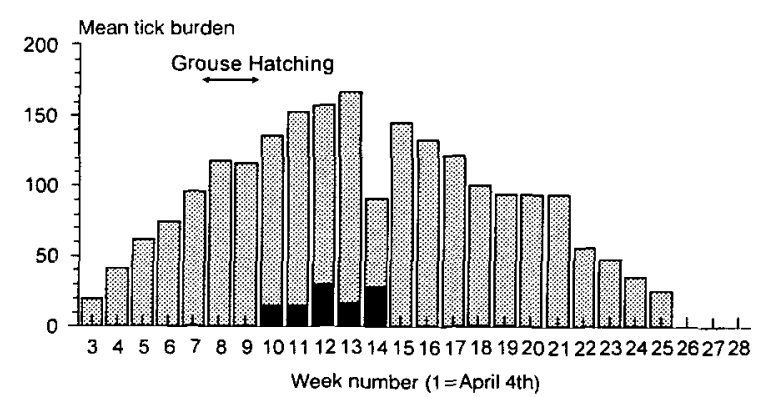

Fig. 3. Mean number of ticks (larvae, nymphs) removed from shot hares (stippled bars) and from live red grouse chicks (solid bars) caught from Lochindorb during the summer of 1993. Grouse chicks do not hatch until about week 9 and are flying after week 14 , grouse will act as hosts after this date but were not sampled.

questing ticks may be bimodal and in others unimodal (Randolph \& Craine, 1995). In general, periods of high tick abundance coincide with the production of young and susceptible hosts (susceptible to both pathogen and vector) and consequently the value of $R_{0}$ will vary seasonally.

Within the louping-ill system, red grouse hatch in late May and early June, coinciding with the period of high abundance of questing ticks, recorded from mountain hares (Fig. 3). Furthermore, immature hosts may be more susceptible to infestation by vectors than adult hosts since a cross-sectional sample of hosts taken when young grouse were 10 weeks of age recorded higher levels of tick infestation in immature grouse than in adults (Hudson, 1986a). This variation between host age classes may be a function of variation in susceptibility to tick infestation with the adults developing resistance. However, there is also the possibility that immature birds have different levels of exposure since they forage for insects in areas where ticks are abundant. The seasonal increase in vectors and the hatching of susceptible hosts will produce a significant seasonal rise in $R_{0}$ and the relative timing of these events could lead to interesting consequences for the dynamics of the disease.

\section{Availability of susceptible hosts : population sinks}

Directly transmitted microparasites that induce a lifelong immune response in their hosts may die out in small populations after the number of susceptible individuals in the host population falls and any remaining susceptibles are protected from infection through herd immunity. Persistence depends on the influx of more susceptible individuals, recruited to the population either by birth or immigration from uninfected areas. Indeed, virulent pathogens can persist within a spatially structured metapopulation when hosts disperse between sub-populations, even when coexistence would not occur at the local population scale (Hassell et al. 1991 ; May \& Nowak, 1994). The pattern of disease flow through the metapopulation will depend specifically on the type of system. Directly transmitted diseases may spread through the host population in a series of waves, but tick-borne diseases are relatively sedentary since ticks are restricted to specific habitats and can survive for a year or more in the absence of hosts. In this respect, tick-borne disease may generate 'host population sinks' by significantly reducing a local group of hosts, leaving a suitable and empty habitat for immigrants, which in turn subsequently become infected and die. Such areas are identified by immigrants as uninhabited suitable habitat, particularly since immigration by susceptibles may occur at a time of year when ticks are not active.

Preliminary population data from estates with louping-ill indicates that the heavy mortality caused by the pathogen is in effect causing population sinks. Such patterns contrast with the areas without louping-ill where winter losses are consistently higher than breeding losses (Hudson, Laurenson \& Reid, unpublished). Levels of infection with loupingill are independent of grouse density and consequently are unlikely to be density dependent and regulate the grouse population. These can be considered true population sinks, not a pseudo-sink where immigrants artificially increase the population above a density dependent regulated density (Watkinson \& Sutherland, 1995).

\section{Host behaviour}

Mating, breeding and foraging behaviour of hosts can increase exposure to infective stages of longlived tick vectors and result in viral persistence within the system. For example, in a number of bird species, males will display on traditional communal 
grounds (leks) producing an aggregated and predictable host population that a tick with an annual cycle could exploit and thus lead to persistence of tick-borne infections. A number of bird groups which breed in a limited nesting habitat will use the same nesting site from one year to the next and thus expose the birds to annually emerging tick vectors. Sea birds often become infected with ticks when using the same nesting ledge or burrows from one year to the next (Danchin, 1992). The ticks must reach and leave the host when the host is at the breeding ledge; any tick that falls off when the bird is at sea will perish.

Suitable nest sites for ground-nesting birds are not usually limiting and precisely the same nest sites are rarely used from one year to the next, even in highly colonial species such as terns. Nevertheless, many ground-nesting species are nidifugous and selectively take their young to invertebrate rich areas to obtain food, as do red grouse (Hudson, 1986a). Areas rich in insect food are frequently damp and consequently suitable for the development and survival of dipteran and arachnid vectors. Ticks tend to be aggregated in areas where the hosts are active and this aggregation will result in local foci of infectious ticks.

\section{Alternative hosts for vector and virus}

In directly transmitted microparasite systems, highly virulent parasites will not have a long-term effect on the size of the host population if the host population is closed (no immigration) since virulent pathogens kill the host rapidly, transmission rate is low and the host population escapes the regulatory role of the parasite. This will not be the case when the parasite infects two hosts and the parasite is more virulent in one host species than the other. In these circumstances, the more resistant host acts as a reservoir, sustaining the parasite and amplifying it, irrespective of the size of the more susceptible host population. The density of the less resistant host does not determine infection rate so a high force of transmission from the reservoir host can lead to localized extinction of the less resistant host species (see review by Begon \& Bowers, 1995).

With vector-borne pathogens, alternative hosts can have three roles. First, as with the directly transmitted two-host microparasites, they may act as reservoir hosts for the virus. Secondly, they may amplify the vector population increasing the ratio of vectors to susceptible hosts and consequently transmission to the susceptible host species. Thirdly, refractory hosts will 'waste' infected tick bites if such tick bites result in no further consequence for the virus. All of these mechanisms may operate in the red grouse-louping-ill system. Sheep are viraemic hosts and will act as reservoir hosts although they can be removed from the system through vaccination and the application of acaracides. Nevertheless, the other non-viraemic mammalian hosts, particularly the mountain hares and red deer, act as hosts for the ticks and increase the ratio of ticks to grouse but they do not amplify the virus and thus 'waste' tick bites. The potential importance of hares to the tick population is illustrated in Fig. 3 where hares carry relatively many more ticks than grouse; with hare densities on our Lochindorb site at approximately 20 pairs $\mathrm{km}^{-2}$ and grouse densities at 6 pairs $\mathrm{km}^{-2}$ this means that hares are probably carrying more than $95 \%$ of the tick population. Hares may have significant consequences for the persistence of the disease as illustrated in Fig. 2. To demonstrate how significant these hosts are to the prevalence of louping-ill requires large-scale manipulative field experiments or a preliminary understanding may be obtained by developing a working model.

The model we develop here examines the dynamics of the pathogen in grouse in relation to the presence of hares. Sheep and other hosts are assumed absent or effectively absent through dipping and vaccination procedures. The basic life cycle of the ticks in relation to the presence of the hare hosts and the flow of the virus through the tick population is illustrated in Fig. $4 a$. The model considers the larval $(L)$, nymph $(N)$ and adult stage $(A)$ of the tick population as either infected $(i)$ or susceptible $(s)$ and the total tick population $(T)$. The grouse population are considered in three classes, the susceptibles $\left(G_{s}\right)$, the infected grouse $\left(G_{i}\right)$ and the immune grouse $\left(G_{z}\right)$, the total grouse population $(G)$ being the sum of these components and the flow of the virus through the grouse population is summarized in Fig. $4 b$. The hares $(H)$ are included as non-viraemic hosts which do not amplify the virus but act as additional hosts for the ticks. The hare population is considered as a constant from one year to the next, an assumption that is not particularly true since hare populations can fluctuate widely.

The per capita birth rate of ticks is $a(\sim 1500$ per adult female) and the per capita death rate of the ticks is $b$, and the various $\beta$ values represent the rate at which the respective tick stages bite the respective host species. Within this initial model we assume mortality rate of the ticks is similar at each stage although once again there can be variation between stages. The density dependence in the grouse and tick populations are represented as $s_{g}$ and $s_{t}$ respectively. The rate at which grouse die from the infection is given as $\alpha$ and they recover to become immune at the rate $\sigma$. The per capita birth and death rates of the grouse are $a_{g}$ and $b_{g}$ respectively. The model assumes no latency and that all infectious ticks biting a grouse result in infection and all susceptible ticks biting an infectious grouse become infected. Later models will examine some of these assumptions in further detail. 

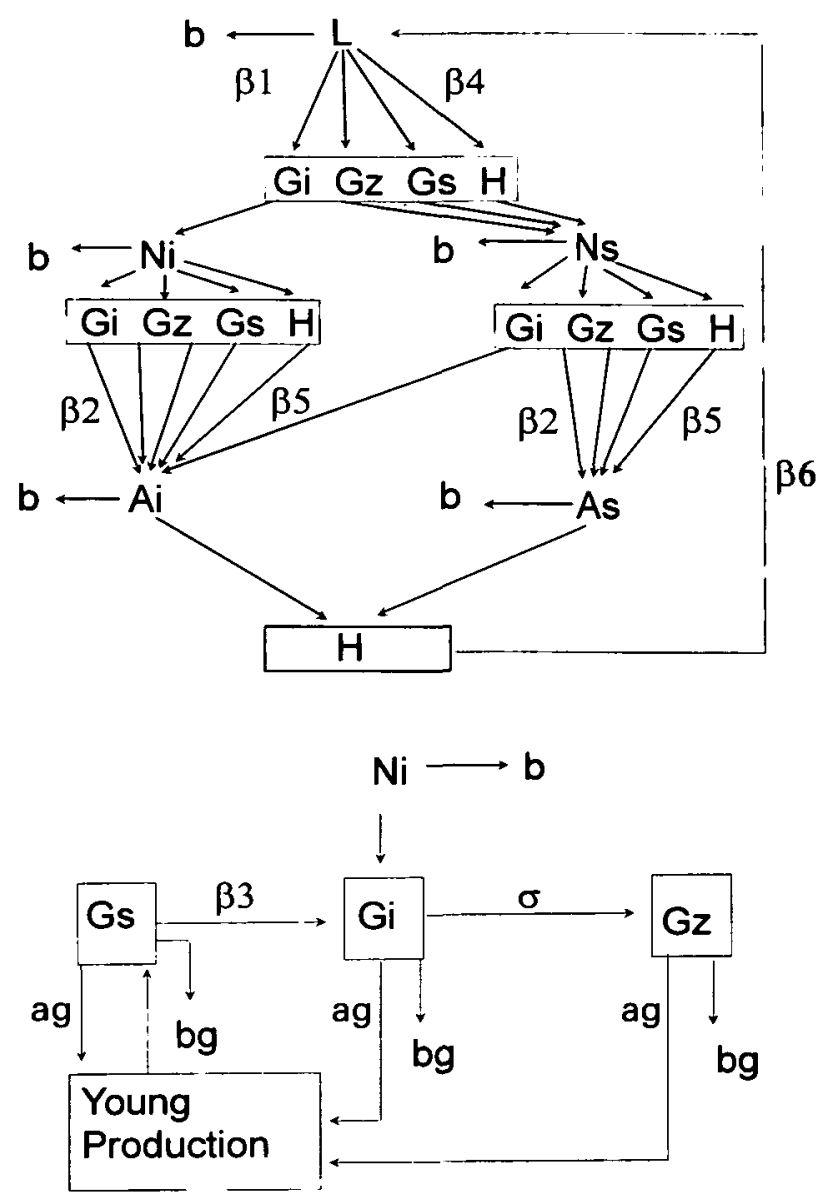

Fig. 4. Flow diagram of the virus through the tick and grouse population: $(a)$ flow of virus through the tick population where the uninfected larvae $(L)$ become infected from infectious grouse $\left(G_{i}\right)$, develop into nymphs $\left(N_{i}\right)$ and then transmit the disease back to susceptible grouse $\left(G_{s}\right)$, note adult ticks $\left(A_{i}\right.$ and $\left.A_{s}\right)$ do not bite grouse, only hares $(H)$ which do not show a viraemic response. (b) Flow of virus through the grouse population, the susceptible grouse $\left(G_{s}\right)$ become infected from a bite by an infectious nymph $\left(N_{i}\right)$ at rate $\beta_{3}$ and then become immune $\left(G_{z}\right)$ at rate $\sigma$, the grouse die at rate $b_{g}$ and produce young at rate $a_{g}$. Note the Beta values show only potential flow of virus, relative values are not known

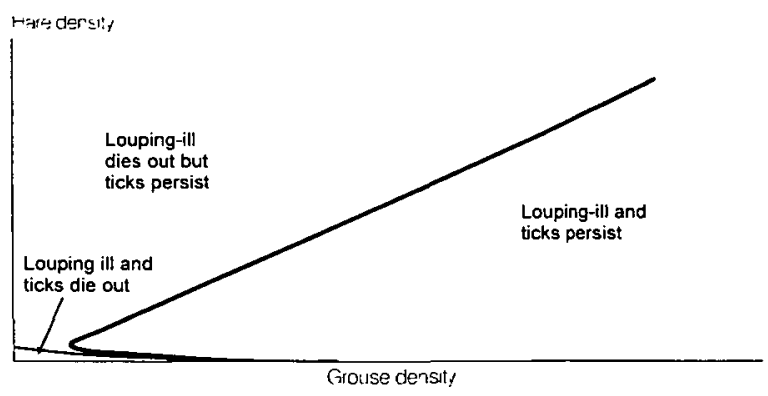

Fig. 5. Phase plane illustrating the potential importance of mountain hares to the maintenance of louping-ill (based on equations 2-7 in text). Hares (or another appreciable mammalian host) are necessary for the life cycle to be sustained and when abundant can sustain the louping-ill virus (after Normal et al. unpublished).
The model is described through a series of coupled differential equations :

$$
\begin{aligned}
& d L / d t=\beta_{6} H\left(a-s_{T} T\right)\left(A_{i}+A_{s}\right) \\
& -\beta_{1} L G-b L-\beta_{4} H L \text {, } \\
& d N_{i} / d t=\beta_{1} G_{i} L-b N_{i}-\beta_{2} N_{i} G-\beta_{5} N_{i} H, \\
& d N_{s} / d t=\beta_{1}(G z+G s) L-b N s \\
& -\beta_{2} N_{s} G+\beta_{4} L H-\beta_{5} N_{8} H \text {, } \\
& d A_{i} / d t=\beta_{2} N_{i} G+\beta_{2} G_{i} N_{s}+\beta_{5} N_{i} H-b A_{i}-\beta_{6} H A_{i} \text {, } \\
& d A_{s} / d t=\beta_{2}\left(G_{z}+G_{s}\right) N_{s}+\beta_{5} N_{s} H-b A_{s}-\beta_{6} H A_{s},(6) \\
& d G_{s} / d t=\left(a_{g}-s_{g} G\right) G-b_{g} G_{s}-\beta_{3} G_{s} N_{i} \text {, } \\
& d G_{i} / d t=\beta_{3} G_{s} N_{i}-\left(b_{g}+\alpha+\sigma\right) G_{i} \text {, } \\
& d G_{z} / d t=\sigma G_{i}-b_{g} G_{z}
\end{aligned}
$$

For the tick population to increase the rate at which they are recruited to each tick class must be greater than their mortality rate, viz:

$$
\begin{aligned}
& \beta_{6} H a\left(\beta_{2} K+\beta_{5} H\right)\left(\beta_{1} K+\beta_{4} H\right) \\
& T_{1} \quad T_{2} \quad T_{3} \\
& >\left(b+\beta_{6} H\right)\left(b+\beta_{1} K+\beta_{4} H\right)\left(b+\beta_{2} K+\beta_{5} H\right) \\
& \begin{array}{lll}
M_{1} & M_{2} & M_{3}
\end{array}
\end{aligned}
$$

where $K$ is the carrying capacity of the grouse population. This is in effect saying that the product of recruitment $\left(T_{1}, T_{2}, T_{3}\right)$ must be greater than the mortality $\left(M_{1}, M_{2}, M_{3}\right)$ and provides us with the estimate of $R_{0}$ for ticks which must be greater than unity for the tick population to establish and increase :

$R_{0(T I C K S)}=\frac{T_{1} T_{2} T_{3}}{M_{1} M_{2} M_{3}}$.

This also provides us with a joint threshold condition, so for any given grouse density we can use this inequality to determine what hare density is needed for tick numbers to increase.

If we consider the persistence of louping-ill virus we obtain a second inequality which is equivalent to $R_{0(D I S E A S E)}$ and another joint threshold curve. Again, for a given grouse density we can use this inequality to determine the hare density, above which the disease will persist. The pathogen will persist when

$\frac{\left(b+\beta_{5} H\right) \Gamma}{\beta_{1} \beta_{3} L^{*}-\beta_{2} \Gamma}<K$,

with

$\beta_{1} \beta_{3} L^{*}>\beta_{2} \Gamma$,

where

$\Gamma=\left(b_{g}+\alpha+\sigma\right)$,

and

$L^{*}=\frac{M_{1} M_{3}\left(T_{1} T_{2} T_{3}-M_{1} M_{2} M_{3}\right)}{s_{t}\left(T_{1} / a\right) T_{2} T_{3}\left(T_{2} T_{3}+M_{1}\left(M_{2}+M_{3}-b\right)\right)}$,

where $s_{t}$ is the density dependence in the tick population. As grouse numbers fall through the effects of the disease, then the virus will die out 
although some hares are needed to keep the tick life cycle going. When there are too many hares present, the hares act as a 'dead end' for infectious ticks to bite and the disease dies out (Fig. 5), an interesting finding explored further by Norman et al. (unpublished). Without the presence of the hares, the tick population and consequently the viral infection cannot persist but when hares are moderately abundant with respect to the grouse density they can sustain both the tick and virus population. Grouse alone can not support the ticks and virus but can do so in conjunction with a significant reservoir host for the vector, even if this host does not produce an appreciable viraemic response.

For a given grouse density, the hare density that would sustain the ticks is sensitive to several of the parameters used in the model but using a range of coarse estimates and an acceptable range of grouse densities then between 5 to 50 hares $\mathrm{km}^{-2}$ are required to sustain both ticks and disease. Two parameters have the largest impact on this threshold hare density; the natural death rate of the ticks $(b)$ and the probability of a tick stage biting a host $\left(\beta_{i}\right)$. In reality, the control of hare numbers below this threshold may not lead to an immediate reduction in disease prevalence since the life cycle takes several years for ticks to complete and so it may take several years for tick and prevalence of louping-ill to be reduced. In the short term, control measures may even result in an increase in the levels of tick infestation on grouse and a consequent rise in prevalence since the removal of the mammalian host may result in more ticks being found on grouse. One of the best approaches to tackling this problem is to undertake field experiments where the hare hosts are controlled and we are currently running a long-term experiment to evaluate the impact of this on the seroprevalence of louping-ill in grouse.

Tick mortality $(b)$ could be increased through two other approaches, first by treating the mammalian hosts with a long lasting acaricide so ticks are killed as they come into contact with the host. This is a tried and tested method with sheep but as yet indirect techniques of applying acaricides to wild animals have not been tried. One method could be to apply acaricide to hare forms. The second method of increasing over-winter mortality of ticks would be to reduce the vegetation that produces a thick, damp matt layer. Ticks survive well when they over-winter in a thick matt layer and Hudson $(1986 b)$ shows that treatment of bracken on grouse moors results in the loss of favoured tick habitat, a reduction in matt and a fall in tick numbers on chicks.

HOST INFECTIOUS PERIOD $\left(1 / \gamma_{1}\right)$

Non-viraemic transmission through co-feeding

One of the most interesting and potentially important mechanisms for virus persistence is non- viraemic transmission, where the virus is transmitted between co-feeding vectors on hosts that do not produce a detectable viraemia (Jones et al. 1987). Non-viraemic transmission has been demonstrated for both Thogoto virus (Orthomyxoviridae) and the flavivirus that causes tick-borne encephalitis (TBE) throughout Europe and Eurasia. Such a process only occurs when ticks are feeding close to one another and is probably enhanced by the presence of tick saliva (Labuda et al. 1993). In epidemiological terms, the largest effects of non-viraemic transmission will be to provide a background force of tick infection, the scale of which depends on the proportion of infectious ticks and particularly infectious larvae the non-viraemic host produces. This will be especially important when the pathogen is virulent and the non-viraemic host acts as a reservoir for both tick and virus.

Within the louping-ill system, we have evidence of louping-ill virus transmission through the mountain hare (Jones et al. unpublished) and some evidence for transmission after sero-conversion. The data presented in Fig. 2 provide some indication of the scale that this could operate at since this compares infection rates in relation to tick abundance between two grouse populations, both with vaccinated sheep populations but one where hares are abundant and one where hares are absent. Infection rate is significantly larger when hares are present, even when tick infestation rates are the same. Such data could well be influenced by other variables but provides a guide for the potential significance of this mechanism.

Some preliminary evidence exists to suggest that non-viraemic transmission may occur in hosts which have sero-converted. In effect, this would mean that a previously viraemic host that had become immune to future infection could still act as an amplifying host through the co-feeding mechanism. This will increase transmission rates and reduce the benefits of herd immunity. This could be important and change the way we view the dynamics of vector-borne pathogens. In the grouse/louping-ill system, the sero-converted grouse and sheep that carry large, aggregated tick burdens could still be acting as sources for virus transmission between ticks and thus producing high rates of virus transmission.

Aggregation of susceptible ticks around an infectious tick is necessary for non-viraemic transmission and the greater this aggregation the greater the proportion of infectious ticks produced and the larger values of $R_{0}$. A suitable formulation to describe such effects would be to replace the mean numbers of ticks per host with an expression that accounts for the extent of aggregation, such as Lloyds mean crowding (Lloyd, 1967) where $s^{2} / m$ is the variance to mean ratio of ticks per host across the population of hosts. If the distribution conforms to a Poisson distribution, then the variance and mean are equal 
and the mean crowding index $\left(m^{*}\right)$ is equal to the true mean $(m)$, but when aggregated the variance exceeds the mean, mean crowding index is larger and transmission and hence $R_{0}$ is increased. Thus in the expression for $R_{0}$ we replace $N_{2} / N_{1}$ with $m^{*}$ where:

$m^{*}=m+\left(\left(s^{2} / m\right)-1\right)$.

\section{Consumption of vectors}

Vectors transmit viruses between hosts principally through taking a blood meal from an infectious host and subsequently biting and infecting a susceptible host. Nevertheless, biting may not be a prerequisite for infection and there is the possibility of hosts becoming infected through other routes. Engorged ticks provide a protein-rich source of food for a number of vertebrate hosts and there is the possibility that hosts may become infected after eating the vectors, either as food or during preening and allopreening of mates. In red grouse, the remains of ticks are frequently recorded in the droppings of immature grouse (Laurenson \& Hudson, unpublished).

Experimental, laboratory studies have found that feeding grouse with louping-ill infected ticks can lead to infection, demonstrating that oral ingestion is a route of infection (Hudson et al. unpublished). Such a transmission route could account for why some seropositive insectivorous hosts record lower levels of tick infestation than expected (e.g. Randolph, 1995).

\section{Latency in vertebrates}

The possibility that there is latency of viral infections in vertebrate hosts has not attracted much attention in laboratory studies since the identification of such a mechanism would be difficult. In essence, the hypothesis is that the virus would be present within a non-viraemic host (and possibly at low levels in a viraemic host after sero-conversion) for a period of time and there would be a possibility of transmission to a vector. In some circumstances, such as when the host is immuno-suppressed through stress or a superinfection, virus levels may rise and increase the probability of infection. In terms of the dynamics of the system, the latency would operate in the same manner as co-feeding but there may be additional seasonal variations according to stress related exposure.

TICK INFECTIOUS PERIOD $\left(1 / \gamma_{2}\right)$

\section{Vertical transmission}

The vertical transmission of viruses from the adult female tick, through the egg, to the larval stage and subsequent stages is a clear adaptation to increase transmission rate and thus lead to persistence of viruses within parasite host systems. This mechanism will not only allow the virus to persist in a system without hosts that produce an appreciable viraemic response but will also produce higher numbers of infectious larvae. The model can be extended to include the effects of trans-ovarial transmission by splitting the larval equations into two so we now consider the situation for infected and susceptible larvae where the probability of a larva being infected is $q$.

$$
\begin{gathered}
d L_{i} / d t=\beta_{6} H q\left(a-s_{T} T\right) A_{i}-b L_{i}-\beta_{1} L_{i} G-\beta_{4} L_{i} H, \\
d L s / d t=\beta_{6} H(1-q)\left(a-s_{T} T\right) A_{i}+\beta_{6} H\left(a-s_{T} T\right) \\
A_{s}-b L_{s}-\beta_{1} L_{s} G-\beta_{4} L_{\delta} H .
\end{gathered}
$$

Trans-ovarial transmission will result in a high proportion of ticks of all stages being infected and consequently higher rates of infection with a higher proportion of immune grouse in the population. At the point when $q=1$ all ticks become infectious and the majority of grouse are immune. In a number of tick-pathogen systems, such as tick-borne encephalitis and lyme disease, trans-ovarial transmission of the pathogen has been identified. However, there is no convincing evidence that the virus that causes louping-ill is transmitted vertically in I. ricinus. We have undertaken some laboratory experiments and shown that no larvae derived from infected female ticks carried the virus; questing larvae from the field were not positive but when we repeated the laboratory experiment with Rhipicephalus appendiculatus vertical transmission was identified.

\section{Tick survival}

The behaviour, survival and fecundity of infected ticks may alter as a direct consequence of carrying a viral infection. This behaviour may act to increase the period of infectiousness or increase the likelihood of transmission but could act in the opposite manner when the infection reduces the survival of infected ticks. For example, babesiosis is known to influence both the survival and fecundity of its tick vectors (Gray, 1982). However, since infection rates are usually low this is unlikely to have any significant regulatory effect on the tick population.

\section{DISCUSSION}

Persistence, within this paper, is taken as the ability of a tick-borne virus to remain active within a host population. The duration of persistence is influenced greatly by the disease mortality (the denominator of $R_{0}$ ) but also by patterns of transmission. Pulses of transmission and the immigration of susceptible hosts may offset high mortality rates and prevent disease fade-out so in this respect we considered persistence as an average value for $R_{0}$ over a time period. The tick-borne pathogens exhibit a wide range of adaptations that may enhance their persistence within a host population. Obviously, stimulating an appreciable viraemic response within the 
vertebrate host can be the principal form of virus multiplication which will result in high transmission and help the virus to persist within the system. However, this paper has highlighted a number of other mechanisms that may permit the pathogen to persist in the absence of viraemic hosts. Some are a function of pathogen biology, some a function of tick biology and some a consequence of the behaviour and ecology of the hosts involved. While we have made some preliminary estimates of the relative importance of these different mechanisms for the louping-ill virus, it is not possible at the current stage to make accurate quantitative comparisons between their relative importance (although this is one of our long-term objectives). Nevertheless, trans-ovarial transmission and non-viraemic transmission through co-feeding are probably the two mechanisms, both influenced by virus biology, that would have the greatest impact on the persistence of a virus.

Trans-ovarial transmission in particular could be a highly effective mechanism for sustaining tick-borne pathogens when few susceptible hosts are available. In the absence of viraemic hosts, the equilibrium of infected ticks would depend on the proportion of female eggs that become infected and the efficacy of subsequent trans-stadial transmission assuming tick survival and fecundity are not influenced by infection. With relatively high rates of vertical transmission, it is possible for most ticks to become infected and for the virus to persist in the absence of a viraemic host population. Moreover, with a highly virulent pathogen this could produce an area of infection in which any immigrating susceptible host would rapidly die.

One interesting feature of trans-ovarial transmission is that some viruses may be transmitted in one tick species but not another and this may vary even between closely related viruses. For example, the virus that causes louping-ill is not vertically transmitted by Ixodes ricinus but is transmitted by the tick Rhipicephalus appendiculatus. The closely related flavivirus that causes tick-borne encephalitis is transmitted vertically within Ixodes ricinus even though the gene sequence of TBE virus only differ from louping-ill virus by a few bases (Shiu, Ayres \& Gould, 1991). This may imply that the structural differences of the virus rather than the vector were important criteria for vertical transmission. More recent comparative studies of the genome of flavivirus indicate that louping-ill virus may only have evolved from TBE virus during the past $200-400$ years and, in moving to other hosts, has lost the ability for transovarial mechanism. Alternatively, the mechanism may operate but the virus kills the tick eggs before the larvae emerge so there is no effective trans-ovarial transmission. Another explanation is that tick hormones may provide cues for the virus to invade the ovaries during egg development and not all viruses respond accordingly (Munderloh $\&$ Kurtti, 1995). Either way, there is clearly a selective advantage for a virus to become vertically transmitted but the relative costs of achieving this may interfere with other aspects of transmission.

Non-viraemic transmission is a second mechanism of infection which may have profound effects on the epidemiology of tick-borne pathogens and allow virus persistence in the absence of viraemic hosts. It would appear that tick saliva is particularly important in facilitating transmission from one tick to another co-feeding tick when the host has an undetectable viraemia, perhaps because of the pharmacological effects of tick saliva on host tissue (Nuttall et al. 1994). Nevertheless, the consequences of this mechanism on the abundance of susceptible host species needs to be examined in relation to the pattern of tick dispersion between host species and the pattern of aggregation on an individual host.

\section{ACKNOWLEDGEMENTS}

We wish to thank the estate owners for their support and permission to work on their land in Yorkshire and Scotland. The field-work would not have been possible without the loyal support of our pointers and setters. Two referees provided valuable and constructive comments. This work was financed by a grant from NERC.

\section{REFERENCES}

ANDERSON, R. M. \& MAY, R. M. (1991). Infectious Disease of Humans. Oxford: OUP.

ARON, J. L. \& MAY, R. M. (1982). The population

dynamics of malaria In Population Dynamics of

Infectious Diseases (ed. Anderson, R. M.), pp. 139-79.

London: Chapman \& Hall.

BEGON, M. \& BOWERS, R. G. (1995). Beyond host-pathogen dynamics. In Ecology of Infectious Diseases in Natural Populations (ed. Grenfell, B. T. \& Dobson, A. P.), pp. 478-509. Cambridge: Cambridge University Press.

DANCHIN, E. (1992). The incidence of the tick parasite Ixodes uriae in Kittiwake Rissa tridactyla colonies in relation to the age of the colony, and a mechanism of infecting new colonies. Ibid 134, 134-41.

DYE, C. \& HASIBEDER, G. (1986). Population dynamics of mosquito-borne disease: effects of flies which bite some people more frequently than others. Transactions of the Royal Society of Tropical Medicine and Hygiene 80, 69-77.

DYE, C. \& Williams, B. G. (1995). Non-linearities in the dynamics of indirectly transmitted infections (or, does having a vector make a difference?). In Ecology of Infectious Diseases in Natural Populations (ed. Grenfell, B. T. \& Dobson, A. P.), pp. 260-279, Cambridge: Cambridge University Press.

GRAY, J. S. (1982). The effects of the piroplasm Babesia bigemina on survival and reproduction of the blue tick, Boophilus decoloratus. Journal of Invertebrate Pathology 39, 413-5. 
haSSEll, M. P., MAY, R. M., PACALA, S. W. \& ChesSON, P. L. (1991). The persistence of host-parasitoid associations in patchy environments. The American Naturalist 138, 568-83.

HUDSON, P. J. (1986a). Red Grouse. The Management of a Wild Gamebird. Fordingbridge, Game Conservancy Trust.

HUDSON, P. J. (1986b). Bracken and ticks on grouse moors in the north of England. In Bracken, Ecology, Land Use and Control Technology. (ed. Smith, R. T. \& Taylor, J. A.), pp. 161-170, Parthenon, Carnforth. Hudson, P. J. (1992). Grouse in Space and Time. Fordingbridge: Game Conservancy Trust.

HUDSON, P. J. \& DOBSON, A. P. (1991). Control of parasites in natural populations: nematodes and virus infections of red grouse. In Bird Population Studies (ed. Perrins, C. M., Lebreton, J.-D. \& Hirons, G. J. M.). Oxford: Oxford University Press.

JONES, L. D., DAVIES, C. R., STEEle, G. M. \& NUTTALl, P. A. (1987). A novel mode of arbovirus transmission involving a nonviraemic host. Science 237, 775-7.

LABUDA, M., JONES, L. D., Williams, T. \& NUTTAlL, P. A. (1993). Enhancement of tick-borne encephalitis virus transmission by tick salivary gland extracts. Medical and Veterinary Entomology 7, 193-6.

LLOYD, M. (1967). Mean crowding. Fournal of Animal Ecology 36, 1-30.

MAY, R. M. \& NOWAK, M. A. (1994). Superinfection, metapopulation dynamics and the evolution of diversity. Fournal of Theoretical Biology 170, 95-114.

MACDONald, G. (1957). The Epidemiology and Control of Malaria. Oxford: Oxford University Press.

MUNDERLOH, U. G. \& KURTTI, T. J. (1995). Cellular and molecular interrelationships between ticks and prokaryotic tick-borne pathogens. Annual Review of Entomology 40, 221-43.

NUTT ALL, P. A., JONES, L. D., LABUDA, M. \& KAUFMAN, W. R. (1994). Adaptations of arboviruses to ticks. fournal of Medical Entomology 31, 1-9.

RANDOLPH, S. E. (1995). Quantifying parameters in the transmission of Babesia microti by the tick Ixodes trianguliceps amongst voles (Clethrionomys glareolus) Parasitology 110, 287-95.

RANDOLPH, S. E. \& CRAINE, N. (1995). A general framework for comparative quantitative studies on the transmission of tick-borne diseases using Lyme borreliosis in Europe as an example. Fournal of Medical Entomology 32, 765-777.

REID, H. W., DUNCAN, J. S., PHILliPS, J. D. P., MOSS, R. \& WATSON, A. (1978). Studies of louping-ill virus (Flavivirus group) in wild red grouse (Lagopus lagopus scoticus). Fournal of Hygiene 81, 321-9.

Ross, R. (1911). The Prevention of Malaria. London: Murray.

SHAW, B. \& REID, H. W. (1981). Immune responses of sheep to louping-ill virus vaccine. Veterinary Record 109, 529-31.

SHIU, S. Y. W., AYRES, M. D. \& GOULD, E. A. (1991). Genomic sequence of the structured proteins of louping-ill: comparative analysis with tick-borne encephalitis virus. Virology 180, 411-15.

SMITH, T. \& KILBORNE, F. L. (1893). Investigations into the nature, causation and prevention of Texas or southern cattle fever. Bulletin No. 1, pp. 301. Washington: USDA, Bureau of Animal Industry. WATKINSON, A. \& SUTHERLAND, w. (1995). Sources, sinks and pseudo-sinks. Fournal of Animal Ecology 64, 126-130. 